\title{
Factors Influencing Nonabsolute Indications for Surgery in Patients With Lower Urinary Tract Symptoms Suggestive of Benign Prostatic Hyperplasia: Analysis Using Causal Bayesian Networks
}

\author{
Myong Kim ${ }^{1}$, Luis Ramirez ${ }^{2}$, Changwon Yoo $^{2}$, Minsoo Choo $^{1}$, Jae-Seung Paick ${ }^{1}$, Seung-June $\mathrm{Oh}^{1}$ \\ ${ }^{1}$ Department of Urology, Seoul National University Hospital, Seoul, Korea \\ ${ }^{2}$ Department of Biostatistics, Florida International University, Miami, FL, USA
}

Purpose: To identify the factors affecting the surgical decisions of experienced physicians when treating patients with lower urinary tract symptoms that are suggestive of benign prostatic hyperplasia (LUTS/BPH).

Methods: Patients with LUTS/BPH treated by two physicians between October 2004 and August 2013 were included in this study. The causal Bayesian network (CBN) model was used to analyze factors influencing the surgical decisions of physicians and the actual performance of surgery. The accuracies of the established CBN models were verified using linear regression (LR) analysis.

Results: A total of 1,108 patients with LUTS/BPH were analyzed. The mean age and total prostate volume (TPV) were 66.2 ( \pm 7.3 , standard deviation) years and $47.3( \pm 25.4) \mathrm{mL}$, respectively. Of the total 1,108 patients, $603(54.4 \%)$ were treated by physician A and 505 (45.6\%) were treated by physician B. Although surgery was recommended to 699 patients (63.1\%), 589 (53.2\%) actually underwent surgery. Our CBN model showed that the TPV $(\mathrm{R}=0.432)$, treating physician $(\mathrm{R}=0.370)$, bladder outlet obstruction (BOO) on urodynamic study (UDS) $(\mathrm{R}=0.324)$, and International Prostate Symptom Score (IPSS) question 3 (intermittency; $\mathrm{R}=0.141$ ) were the factors directly influencing the surgical decision. The transition zone volume $(\mathrm{R}=0.396)$, treating physician $(\mathrm{R}=0.340)$, and $\mathrm{BOO}(\mathrm{R}=0.300)$ directly affected the performance of surgery. Compared to the LR model, the area under the receiver operating characteristic curve of the CBN surgical decision model was slightly compromised $(0.803$ vs. $0.847, \mathrm{P}<0.001)$, whereas that of the actual performance of surgery model was similar $(0.801$ vs. 0.820 , $\mathrm{P}=0.063)$ to the LR model.

Conclusions: The TPV, treating physician, BOO on UDS, and the IPSS item of intermittency were factors that directly influenced decision-making in physicians treating patients with LUTS/BPH.

Keywords: Bayes Theorem; Decision Support Techniques; Decision Making, Computer-Assisted; Prostatic Hyperplasia; Urodynamics

- Grant Support: This study was supported by grant No. 04-2010-1080 from the Seoul National University Hospital Research Fund.

- Research Ethics: This stydy was approved by Institutional Review Board of Seoul National University Hospital (SNUH) (No. H 1106-066-366).

- Conflict of Interest: No potential conflict of interest relevant to this article was reported.

\section{INTRODUCTION}

Recent international guidelines on lower urinary tract symp- toms that are suggestive of benign prostatic hyperplasia (LUTS/ $\mathrm{BPH}$ ) recommend that surgery is indicated when complications are present $[1,2]$. However, most patients with LUTS/BPH are

Corresponding author: Seung-June Oh (iD http://orcid.org/0000-0002-0322-3539 Department of Urology, Seoul National University Hospital, 101 Daehak-ro, Jongno-gu, Seoul 110-744, Korea

E-mail: sjo@snu.ac.kr / Tel: +82-2-2072-2406 / Fax: +82-2-742-4665

Submitted: August 22, 2014 / Accepted after revision: October 31, 2014

(c) (1) $\$$ This is an Open Access article distributed under the terms of the Cre(c) commons.org/licenses/by-nc/3.0/) which permits unrestricted non-commercial use, distribution, and reproduction in any medium, provided the original work is properly cited. 
not absolutely indicated for surgery [3]. Hence, the physician must decide the best initial treatment (including whether to perform surgery) on a case-by-case basis according to clinical conditions.

For that decision, numerous clinical parameters must be considered, such as: LUTS type and severity; voiding pattern identified by a bladder diary; response to oral medication; prostate size; and uroflowmetry (UFM) parameters, including postvoid residual (PVR), endoscopic findings, or urodynamic study (UDS) results $[1,2]$. The decision is also influenced by patientrelated factors, such as age, patient preference, willingness to accept surgery-associated complications (e.g., retrograde ejaculation), comorbidities, current medications (such as anticoagulants), socioeconomic status, medical insurance, personal schedule, and geographic accessibility to a clinic [1,2].

The analysis of the clinical decisions made by experienced physicians in the management of LUTS/BPH is important because the related factors related to the decision made can be integrated, and can thus provide a new level of evidence for the rational management of LUTS/BPH [4]. However, few studies have investigated LUTS/BPH management decisions [5]. Since many factors require consideration, some of which have covariability (interaction between parameters) [6], the associations between influencing factors and surgical decisions are complex and largely unclear. To overcome these problems, the causal Bayesian network (CBN) model has emerged as a more advanced alternative to conventional statistical models in the medical field [7]. The benefit of this model is that it can be used to visualize the interaction of cause-effect relationships and can eliminate indirect causes of events [8]. Therefore, this novel model is suitable for analyzing clinical decisions and establishing a clinical decision support system in the medical field [9].

We hypothesized that the CBN model can analyze the surgical decisions of physicians for patients with LUTS/BPH and can determine some direct causes of the decisions without compromised predictive values. In the present study, we aimed to identify the factors directly influencing the surgical decisions of experienced physicians and the actual performance of the surgery using the CBN model.

\section{MATERIALS AND METHODS}

The Institutional Review Board of Seoul National University Hospital (SNUH) approved the present study (No. H 1106-066366). The inclusion criteria were male patients with LUTS/BPH and age of $\geq 45$ years. Patients with a history of genitourinary surgery, genitourinary malignancy, pelvic radiation therapy, current urinary tract infection (UTI), urethral stricture, interstitial cystitis, neuropathy suggestive of neurogenic bladder, and incomplete evaluations were excluded from the analysis. Among them, patients who were absolutely indicated for surgery by the international treatment guidelines [1,2], including those with refractory retention, recurrent gross hematuria, bladder stone, recurrent UTI, or renal insufficiency, were also excluded, as our intent was to analyze patients with LUTS/BPH who had only relative indicators for surgery.

The following UDS results and clinical parameters were collected from our institution's Urodynamic Database Registry and Electronic Medical Records system: history, physical examination, International Prostate Symptom Score (IPSS) [10], UFM parameters (including PVR), prostate-specific antigen (PSA) level, and prostate size as measured by transrectal ultrasonography. Moreover, whether the physician recommended the surgery and whether the operation was actually performed were also reviewed. If the decision to perform surgery and actual performance of surgery differed, the reason was identified. All UDS (UD-2000, Medical Measurement Systems B.V., Enschede, the Netherlands) were performed according to the recommendations of the International Continence Society [11,12]. The bladder outlet obstruction (BOO) index was defined as detrusor pressure at the maximal flow rate (PdetQmax) $-2 \times$ maximum flow rate ( $\mathrm{Qmax})$, and the presence of $\mathrm{BOO}$ was defined by a BOO index of $\geq 40$ [13].

Fig. 1 shows a simple CBN model. In that model, events A and D are in the causal Markov condition [14]. Since event D is not directly influenced by event A, the conditions of events $\mathrm{B}$ and $C$ directly affect event $D$, whereas information about event A is not needed. The causal Markov condition can be visually

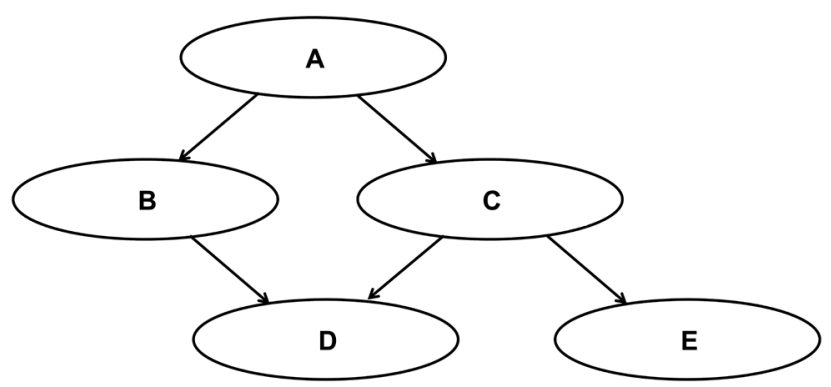

Fig. 1. A simple causal Bayesian network model that can visualize the causal Markov conditions in male patients with lower urinary tract symptoms. 
identified in the $\mathrm{CBN}$, which has a relationship of two or more degrees between nodes. In Fig. 1, events B and C are in a firstdegree relationship with event $\mathrm{D}$, meaning that events $\mathrm{B}$ and $\mathrm{C}$ are factors directly influencing event $\mathrm{D}$. The probability of event $\mathrm{D}$ occurring can be expressed as $P$ (event $D$ event $B$, event $C$ ). The joint probability of multiple events $\left(X_{1}, X_{2}, \ldots, X_{n}\right)$ in specific situations $(K)$ can be calculated as follows:

$$
P\left(X_{1}, X_{2}, \ldots, X_{n} \mid K\right)=\prod_{i=1}^{n} P\left(X_{i} \mid \pi_{i}, K\right)
$$

where $\pi_{i}$ indicates a joint state of the parents of $X_{i}$, and $K$ denotes background knowledge [8].

The CBN model was used to analyze the decision of physicians to perform surgery in patients with relative indications for the surgery. The clinical parameters considered to be causal factors contributing to the performance of surgery include the treating physician (two physicians masked as A and B), age, Qmax, PVR, PSA level, total prostate volume (TPV), transition zone volume (TZV), BOO on UDS, and IPSS (each individual score for questions 1 to 7 , quality of life question [IPSS-QoL], emptying sum score [IPSS-emptying, sum of questions 1, 3, 5, and 6], storage sum score [IPSS-storage, sum of questions 2, 4, and 7], and total sum score [IPSS-total, sum of questions 1 to 7]). All causative variables were categorized into three groups by distribution (cutoff points: mean $\pm 1 / 2 \times$ standard deviation [SD]). Other patient-related factors (e.g., comorbidity, socioeconomic status) were excluded from the analysis. In the CBN models, nodes showing a first-degree relationship with surgical decisionmaking were identified as directly influencing factors. Other nodes were considered to have a causal Markov condition. The directional nature of each tie was ignored. The weight of each directly influencing factor was estimated using the Spearman's correlation test.

Two CBN models were established to identify the direct cause(s) of two major events: (1) the surgical decision of the physician (surgical decision-CBN model); and (2) the actual performance of LUTS/BPH surgery (actual operation-CBN model). For the surgical decision-CBN model, the aforementioned causative factors and whether the physician recommended surgery were evaluated. In the actual operation-CBN model, causative factors and whether the operation was actually performed were evaluated.

The accuracy of these two models was verified by the predictive values obtained on a backward stepwise linear regression (LR) analysis [15]. The CBN models were established using Banjo ver. 2.2.0 (Duke University, Durham, NC, USA). The mode of the CBN models was a high-scoring network that limited the number of parents to five and ran the analysis for up to 6 hours. To compare accuracy, the comparison of the receiver operating characteristic (ROC) curves described by DeLong et al. [16] was applied using Medcalc ver. 12.4.0 (MedCalc Software, Ostend, Belgium). Other descriptive statistics were processed using PASW Statistics ver. 18.0 (SPSS Inc., Chicago, IL, USA). Values with $\mathrm{P}<0.05$ were considered significant.

\section{RESULTS}

From October 2004 to August 2013, a total of 2,492 patients aged $\geq 45$ years with LUTS/BPH were identified from among 10,184 cases in the SNUH Urodynamic Database Registry. Among them, 1,299 patients were treated by two physicians (S.J.O. and J.S.P.). Of the 1,299 patients, 120 (9.3\%) were excluded according to the exclusion criteria mentioned above; thus, 1,179 subjects remained. Among them, 71 (6.0\%) were absolutely indicated for surgery $[1,2]$, and the remaining 1,108 patients with LUTS/BPH had relative indicators for surgery.

The patient demographics are shown in Table 1. The mean age was $66.2( \pm 7.3, \mathrm{SD})$ years. The TPV and PSA were 47.3 $( \pm 25.4) \mathrm{mL}$ and $2.47( \pm 3.19) \mathrm{ng} / \mathrm{mL}$, respectively. IPSS-total and IPSS-QoL scores were $18.0( \pm 7.8)$ and $4.0( \pm 1.2)$, respectively. The mean $\mathrm{BOO}$ index was $33.8( \pm 24.5)$, and 331 patients (29.9\%) were classified as having BOO. Of the 1,108 patients, 603 (54.4\%) were treated by physician A and 505 (45.6\%) were treated by physician B. Although surgery was recommended for 699 patients (63.1\%), 589 (53.2\%) actually underwent surgery. Details of the differences between surgical decisions and performed surgeries are shown in Table 1.

\section{Which Parameters Influenced the Surgical Decision? (Surgical Decision Model)}

The best CBN structure for the surgical decision-CBN model is shown in Fig. 2. The treating physician, TPV, response to IPSS question 3 (intermittency), and BOO on UDS had first-degree relationships with the physician's decision. Therefore, those four factors were selected as the factors directly influencing the physicians' surgical decisions. The correlation coefficient was the highest for TPV $(\mathrm{R}=0.432, \mathrm{P}<0.001)$, followed by treating physician $(\mathrm{R}=0.370, \mathrm{P}<0.001), \mathrm{BOO}$ on UDS $(\mathrm{R}=0.324, \mathrm{P}<0.001)$, and response to IPSS question $3(\mathrm{R}=0.141, \mathrm{P}<0.001)$. The area under the receiver operating characteristic curve (AUROC) of the surgical decision-CBN model was 0.803 , which was compromised 
Table 1. Characteristics of the 1,108 included patients

\begin{tabular}{|c|c|}
\hline Characteristic & Value \\
\hline Age (yr) & $65.9 \pm 7.2$ \\
\hline \multicolumn{2}{|l|}{ Prostate volume (mL) } \\
\hline Total prostate volume & $47.3 \pm 25.4$ \\
\hline Transition zone volume & $22.8 \pm 18.7$ \\
\hline Prostate-specific antigen (ng/mL) & $2.47 \pm 3.19$ \\
\hline \multicolumn{2}{|l|}{ International Prostatic Symptom Score (IPSS) } \\
\hline IPSS-total & $18.0 \pm 7.8$ \\
\hline IPSS-emptying & $10.9 \pm 5.5$ \\
\hline IPSS-storage & $7.1 \pm 3.5$ \\
\hline IPSS-quality of life & $4.0 \pm 1.2$ \\
\hline \multicolumn{2}{|l|}{ Uroflowmetry parameters } \\
\hline Maximum flow rate (mL/sec) & $11.7 \pm 4.9$ \\
\hline Postvoid residual volume (mL) & $55.7 \pm 76.7$ \\
\hline \multicolumn{2}{|l|}{ Urodynamic study parameters } \\
\hline Maximal urethral closure pressure $\left(\mathrm{cm} \mathrm{H}_{2} \mathrm{O}\right)$ & $75.0 \pm 26.4$ \\
\hline Functional urethral length (mm) & $71.8 \pm 21.7$ \\
\hline First desire $(\mathrm{mL})$ & $199.9 \pm 89.7$ \\
\hline Normal desire (mL) & $283.9 \pm 108.0$ \\
\hline Strong desire $(\mathrm{mL})$ & $374.3 \pm 108.9$ \\
\hline Compliance $\left(\mathrm{mL} / \mathrm{cm} \mathrm{H}_{2} \mathrm{O}\right)$ & $65.9 \pm 49.9$ \\
\hline $\operatorname{PdetQmax}\left(\mathrm{cm} \mathrm{H}_{2} \mathrm{O}\right)$ & $51.4 \pm 21.0$ \\
\hline Opening pressure $\left(\mathrm{cm} \mathrm{H}_{2} \mathrm{O}\right)$ & $52.2 \pm 24.9$ \\
\hline Bladder outlet obstruction index & $33.8 \pm 24.5$ \\
\hline \multicolumn{2}{|l|}{ Treated by } \\
\hline Physician A & $603(54.4)$ \\
\hline Physician B & $505(45.6)$ \\
\hline Recommended to do surgery & $699(63.1)$ \\
\hline Actually received surgery & $589(53.2)$ \\
\hline Transurethral prostatectomy & $8(0.7)$ \\
\hline Open prostatectomy & $3(0.3)$ \\
\hline Holmium laser enucleation of the prostate & $402(36.3)$ \\
\hline Photoselective vaporization of prostate & $158(14.3)$ \\
\hline Transurethral incision & $13(1.2)$ \\
\hline \multicolumn{2}{|l|}{$\begin{array}{l}\text { Details in the difference between the surgical } \\
\text { decision and actual operation }\end{array}$} \\
\hline Not recommended surgery but received & 3 \\
\hline Do not want oral medication & $3(100)$ \\
\hline Recommended surgery but not received & 113 \\
\hline Underlying risks for anesthesia & $6(5.3)$ \\
\hline Economic problem & $2(1.8)$ \\
\hline Fear for operation (pain, complication, etc.) & $37(32.7)$ \\
\hline Personal problem (other plan, etc.) & $13(11.5)$ \\
\hline WeIl-controlled by oral medication & $43(38.1)$ \\
\hline Unidetified reasons & $12(10.6)$ \\
\hline
\end{tabular}

Values are presented as mean \pm standard deviation or number (\%).

IPSS-total, sum of questions 1 to 7; IPSS-emptying, sum of questions 1, 3, 5 and 6; IPSS-storage, sum of questions 2, 4, and 7; IPSS-QoL, quality of life question score; PdetQmax, detrusor pressure at maximum flow rate.

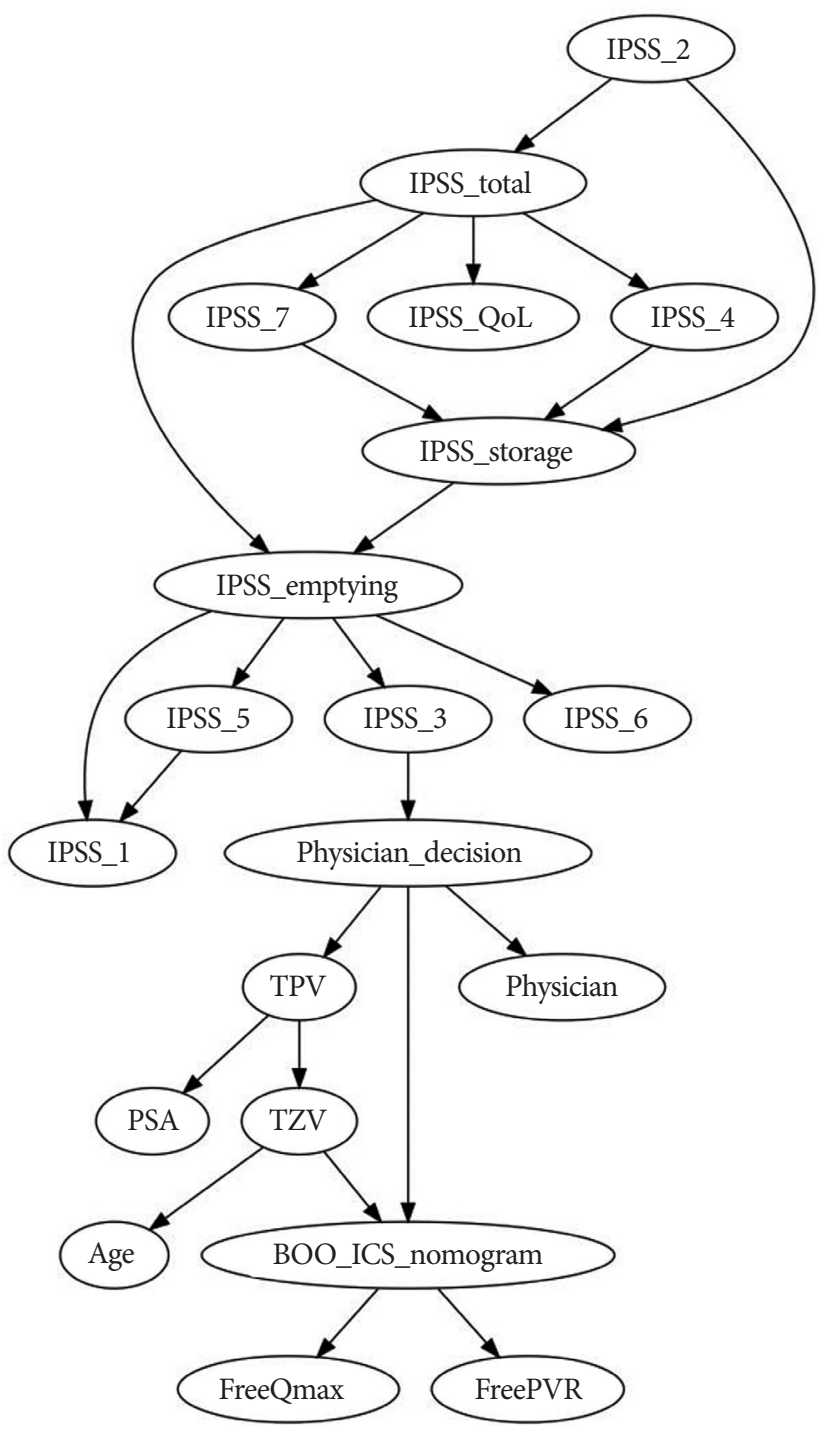

Fig. 2. A causal Bayesian network model for surgical decisions (Surgical Decision-CBN Model), which indicates the parameters that directly influenced the physician's surgical decision. The CBN models were established using Banjo ver. 2.2.0 (Duke University, Durham, NC, USA). IPSS, International Prostate Symptom Score; IPSS-total, sum of questions 1 to 7; IPSS-emptying, sum of questions 1, 3, 5 and 6; IPSS-storage, sum of questions 2, 4, and 7; IPSS-QoL, quality of life question score; BOO_ICS_nomogram, bladder outlet obstruction index; TPV, total prostate volume; TZV, transition zone volume; PSA, prostate-specific antigen; FreeQmax, maximum flow rate on uroflowmetry; FreePVR, postvoid residual.

compared to that of the LR models with the same parameters (surgical decision-LR model; $\mathrm{P}<0.001$ ) (Fig. 3A). The results of the surgical decision-LR model are shown in Table 2. 

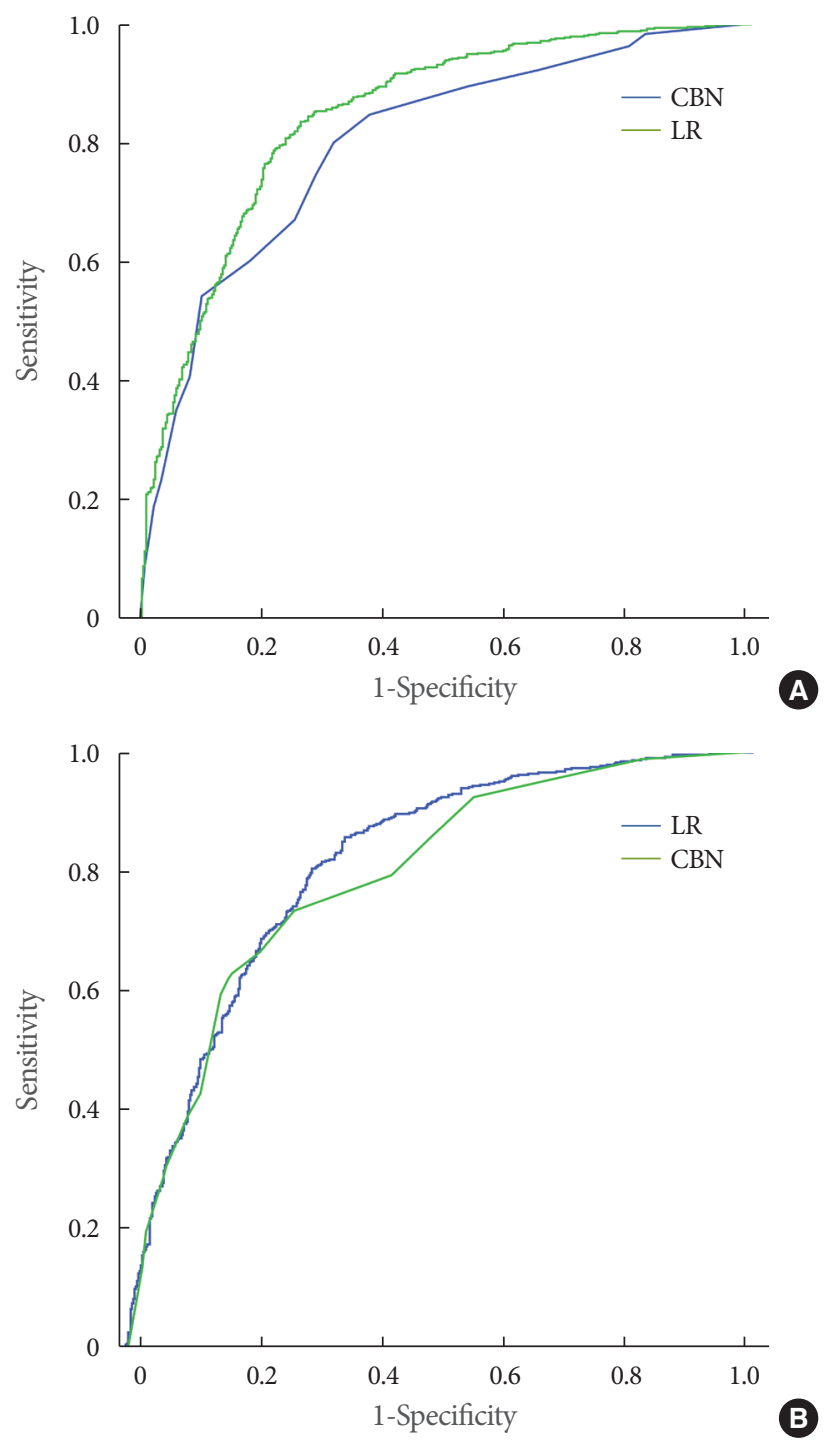

Fig. 3. Comparison of receiver operating characteristic (ROC) curves between the causal Bayesian network (CBN) models and linear regression (LR) models. (A) Surgical decision model $(n=1,108)$. Area under the ROC curve: surgical decision-CBN model, 0.803 (95\% confidence interval [CI], 0.779-0.826); surgical decision-LR model, 0.847 (95\% CI, 0.824-0.868). Pairwise comparison of ROC curves: Difference between areas, 0.0438 ( $\mathrm{P}<0.001$; 95\% CI, 0.01920.0683). (B) Actual surgery model $(n=1,108)$. Area under the ROC curve: actual surgery-CBN model, 0.801 (95\% CI, 0.777-0.824); actual surgery-LR model, 0.820 (95\% CI, 0.796-0.842). Pairwise comparison of the ROC curves: Difference between areas: 0.0184 ( $\mathrm{P}=0.063 ; 95 \% \mathrm{CI},-0.001$ to 0.038$)$.

\section{Parameters Directly Affecting Actual Performance of Surgery (Actual Surgery Model)}

Fig. 4 shows the best $\mathrm{CBN}$ structure for the actual surgery-CBN model. The treating physician, TZV, and BOO on UDS showed a

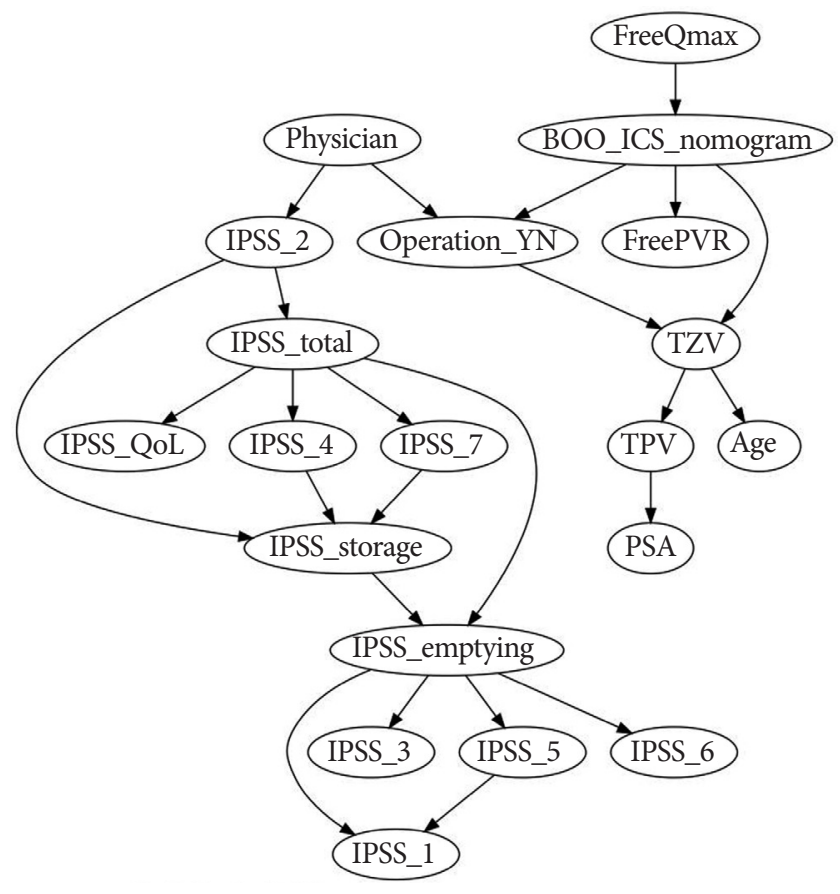

Fig. 4. A causal Bayesian network model for actual performance of surgery (actual surgery-CBN model), which indicates the parameters that directly affected actual surgical performance. IPSS, International Prostate Symptom Score; IPSS-total, sum of question 1 to 7; IPSS-emptying, sum of questions 1, 3, 5 and 6; IPSSstorage, sum of questions 2, 4, and 7; IPSS-QoL, quality of life question score; BOO_ICS_nomogram, bladder outlet obstruction index; TPV, total prostate volume; TZV, transition zone volume; PSA, prostate-specific antigen; FreeQmax, maximum flow rate on uroflowmetry; FreePVR, postvoid residual.

first-degree relationship with the actual surgery. Therefore, those three factors were selected as the factors directly influencing actual surgery. The correlation coefficient was the highest for TZV $(\mathrm{R}=0.396, \mathrm{P}<0.001)$, followed by treating physician $(\mathrm{R}=0.340$, $\mathrm{P}<0.001)$ and $\mathrm{BOO}$ on UDS $(\mathrm{R}=0.300, \mathrm{P}<0.001)$. The AUROC of the actual surgery-CBN model was 0.801 , which was not statistically different from that of the actual surgery-LR model with the same parameters $(\mathrm{P}=0.063)$ (Fig. 3B). The results of the actual surgery-LR model are shown in Table 2.

\section{DISCUSSION}

Although a large number of studies have been published over several decades, there is very often insufficient evidence based on which clear statements about "the right treatment for every patient" can be made [4]. Moreover, changes in other modalities, such as oral medication, can cause changes in trends for the sur- 
Table 2. Multiple linear regression (LR) models with the same parameters as the causal Bayesian network models (only the parameters enrolled within the models are presented)

\begin{tabular}{|c|c|c|c|c|c|c|c|}
\hline & \multicolumn{2}{|c|}{ Unstandardized coefficients } & \multirow{2}{*}{$\frac{\text { Standardized coefficients }}{\beta}$} & \multirow{2}{*}{$\mathrm{t}$} & \multirow{2}{*}{ P-value } & \multicolumn{2}{|c|}{ Collinearity statistics } \\
\hline & $\mathrm{B}$ & SE & & & & Tolerance & VIF \\
\hline \multicolumn{8}{|c|}{ Surgical decision-LR model $(\mathrm{n}=1,108)$} \\
\hline (Constant) & -0.408 & 0.137 & & -2.986 & 0.003 & & \\
\hline Age & 0.005 & 0.002 & 0.073 & 2.796 & 0.005 & 0.899 & 1.113 \\
\hline Qmax & -0.013 & 0.003 & -0.132 & -5.005 & $<0.001$ & 0.891 & 1.123 \\
\hline TPV & 0.004 & 0.001 & 0.227 & 8.294 & $<0.001$ & 0.824 & 1.214 \\
\hline IPSS Q5 & 0.030 & 0.008 & 0.100 & 3.630 & $<0.001$ & 0.806 & 1.240 \\
\hline IPSS Q7 & -0.025 & 0.010 & -0.066 & -2.438 & 0.015 & 0.833 & 1.201 \\
\hline IPSS Q8 & 0.022 & 0.012 & 0.054 & 1.886 & 0.060 & 0.739 & 1.353 \\
\hline BOO on UDS & 0.203 & 0.029 & 0.193 & 6.971 & $<0.001$ & 0.807 & 1.239 \\
\hline Physician (B) & 0.323 & 0.024 & 0.333 & 13.258 & $<0.001$ & 0.975 & 1.025 \\
\hline \multicolumn{8}{|c|}{ Actual surgery-LR model $(\mathrm{n}=1,108)$} \\
\hline (Constant) & -0.563 & 0.145 & & -3.889 & $<0.001$ & & \\
\hline Age & 0.005 & 0.002 & 0.071 & 2.628 & 0.009 & 0.899 & 1.113 \\
\hline Qmax & -0.012 & 0.003 & -0.114 & -4.241 & $<0.001$ & 0.891 & 1.123 \\
\hline TPV & 0.005 & 0.001 & 0.235 & 8.374 & $<0.001$ & 0.824 & 1.214 \\
\hline IPSS Q5 & 0.027 & 0.009 & 0.086 & 3.032 & 0.002 & 0.806 & 1.240 \\
\hline IPSS Q7 & -0.026 & 0.011 & -0.067 & -2.411 & 0.016 & 0.833 & 1.201 \\
\hline IPSS Q8 & 0.041 & 0.013 & 0.096 & 3.239 & 0.001 & 0.739 & 1.353 \\
\hline BOO on UDS & 0.187 & 0.031 & 0.172 & 6.070 & $<0.001$ & 0.807 & 1.239 \\
\hline Physician (B) & 0.305 & 0.026 & 0.304 & 11.800 & $<0.001$ & 0.975 & 1.025 \\
\hline
\end{tabular}

SE, standard error; t, T score; VIF, variance inflation factor; Qmax, maximum flow rate on uroflowmetry, TPV; total prostate volume; IPSS, International Prostate Symptom Score; BOO, bladder outlet obstruction; UDS, urodynamic study.

gical treatment of LUTS/BPH [17]. The treatment outcomes depend on the success standard. For instance, there has been controversy over the usefulness of UDS in preoperative LUTS/BPH evaluation. However, Javle et al. [18] reported that treatment failure occurred in $100 \%$ of patients in a urodynamically unobstructed subgroup after transurethral prostatectomy (TURP); therefore, UDS is helpful for ruling out a subgroup of patients who would not benefit. On the other hand, Gotoh et al. [19] reported that patients with minimal urodynamic obstruction also have a good outcome; therefore, such patients should not necessarily be considered contraindicated for surgery. However, those two studies had different definitions of treatment success - the former strictly defined success as 50\% improvement in IPSS, Qmax, and PVR [19], whereas the latter defined it as fair, good, or excellent on a subjective satisfaction scale (full scale: poor/ fair/good/excellent) [19]. It is already known that UFM parameters and voiding symptoms are not well correlated [20].
The large numbers of published studies providing conflicting evidence as well as numerous other issues surrounding the acceptance of such evidence become barriers to using research evidence in daily practice [21]. However, analyzing the logic of experts can provide a new level of evidence for the rational management of LUTS/BPH [4]. Our CBN results demonstrated that TPV $(\mathrm{R}=0.432)$, treating physician $(\mathrm{R}=0.370)$, BOO on UDS $(\mathrm{R}=0.324)$, and response to IPSS question $3(\mathrm{R}=0.141)$ directly influenced the surgical decision for patients with LUTS/BPH (Fig. 2). Seki et al. [22] studied the prognostic factors for outcomes after TURP and concluded that Schäfer's grade (grade of urodynamic obstruction [23]) and detrusor overactivity in UDS were related to outcomes. However, prostate volume, which was an important surgical decision-making factor in the present study, was not considered in the model of Seki et al. [22]. The consideration of all proven decision-making factors in a prognostic model is assumed to be more rational. 
It is interesting that response to IPSS question 3 can represent all other IPSS domains as a factor directly influencing physicians' surgical decisions (Fig. 2). Our surgical decision-CBN model (Fig. 2) demonstrated that all domains of the IPSS are clustered and that they converge on IPSS question 3. These findings suggest that each IPSS domain had covariability and did not independently affect the surgical decision. This relationship enabled the ruling out of other IPSS domains as influencing factors on surgical decisions. In the actual surgery-CBN model, similar factors (TZV $[\mathrm{R}=0.396]$, physician $[\mathrm{R}=0.340]$, and $\mathrm{BOO}$ on UDS $[R=0.300]$ ) except for response to IPSS question 3 affected the actual performance of surgery (Fig. 4), although there were some discrepancies between the surgical decision and actual performance of surgery (116 of 1,108 patients [10.5\%]; Table 1 ). However, the patient-related factors, such as patient's preference, willingness to accept complications, comorbidities, socioeconomic status, or hospital accessibility, were not fully considered in this model due to the lack of information.

The predictive performance of our surgical decision-CBN model is slightly compromised compared to that of the surgical decision-LR model (AUROC $=0.803$ vs. 0.847 , respectively; $\mathrm{P}<0.001$ ) (Fig. 3A). A counterbalance of the cause-and-effect relationships arising from various logical processes of the physicians' clinical decisions was postulated as the cause of those findings. When the surgical decision was analyzed for each physician separately, the directly influencing factors differed (physician A: TZV [ $\mathrm{R}=0.482$ ], BOO on UDS [ $\mathrm{R}=0.469$ ], PVR [ $R=0.242]$, and response to IPSS question 3 [ $R=0.141]$; physician B: TZV [R=0.239]; figures not presented). The result that discrete models by individual physicians had equivalent predictive performances to LR models support that deduction. When pursuing logic integration as a new level of evidence, such as in the trial of Stoevelaar et al. [4], the latent risk of the counterbalance of cause-and-effect relationships between logics should be considered. Although the two physicians in the present study used different logic methods to make surgical decisions, they did not violate the statements regarding the relative surgical indicators of LUTS/BPH (nonresponders to medical treatment or those who do not want medical therapy but request active treatment) based on international recommendations [1,2].

We did not analyze which patients treated under the specific logic of a physician had better treatment outcomes. We believe that a definitive analysis of the treatment outcomes of patients treated by the different logics is a very important subject of a further study. Moreover, because our decision models are based on the experiences of only two physicians, the results may not be generalizable. However, it is important to note that the interaction of cause-effect relationships impacting surgical decisions can be visualized using a CBN model. By selecting only the directly influencing factors, two models (surgical decision and actual surgery-CBN models) demonstrated moderate predictive performance (AUROC $=0.803$ and 0.801, respectively). Based on this information and some additional data, a well-formed clinical decision support system for LUTS/BPH can eventually be established, as in other medical fields [9]. However, the CBN model of this study has some limitations, as follows: indirect weighting of contributors; ignoring the directional natures of each tie due to the limitations of the cross-sectional database; and conversion of continuously valued clinical parameters to categorical values. Therefore, an improved model is needed.

The CBN model can visualize the interaction of cause-effect relationships impacting physicians' decisions for LUTS/BPH patients. Our CBN model shows that TPV, treating physician, $\mathrm{BOO}$ on UDS, and response to IPSS question 3 were factors directly influencing surgical decisions made by physicians regarding patients with LUTS/BPH. Among these, TPV was the most important factor for surgical decisions.

\section{ACKNOWLEDGEMENTS}

We would like to thank Yu-Kyung Lee who assisted with database management.

\section{REFERENCES}

1. Oelke M, Bachmann A, Descazeaud A, Emberton M, Gravas S, Michel MC, et al. EAU guidelines on the treatment and follow-up of non-neurogenic male lower urinary tract symptoms including benign prostatic obstruction. Eur Urol 2013;64:118-40.

2. McVary KT, Roehrborn CG, Avins AL, Barry MJ, Bruskewitz RC, Donnell RF, et al. Update on AUA guideline on the management of benign prostatic hyperplasia. J Urol 2011;185:1793-803 .

3. Gonzalez RR, Kaplan SA. First-line treatment for symptomatic benign prostatic hyperplasia: is there a particular patient profile for a particular treatment? World J Urol 2006;24:360-6.

4. Stoevelaar HJ, McDonnell J, Bosch JL, Kahan JP; European Panel on the Appropriate Treatment of BPH. Lower urinary tract symptoms suggestive of benign prostatic obstruction: how can clinical expertise contribute to rational management? Eur Urol 2001;39 Suppl 3:13-9. 
5. Chicharro Molero JA, Burgos Rodriguez R, Sanchez Cruz JJ. Analysis of the clinical decision in the initial management of BPH. Actas Urol Esp 1998;22:103-10.

6. Tu JV. Advantages and disadvantages of using artificial neural networks versus logistic regression for predicting medical outcomes. J Clin Epidemiol 1996;49:1225-31.

7. Nikovski D. Constructing Bayesian networks for medical diagnosis from incomplete and partially correct statistics. IEEE Trans Knowl Data Eng 2000;12:509-16.

8. Pearl J. Bayesian inference. In: Brachman RJ, editor. Probabilistic reasoning in intelligent systems: networks of plausible inference. 2nd ed. San Francisco: Morgan Kaufmann Publisher; 1988. p. 29-75.

9. Kong G, Xu DL, Yang JB. Clinical decision support systems: a review on knowledge representation and inference under uncertainties. Int J Comput Intell Syst 2008;1:159-67.

10. Choi HR, Chung WS, Shim BS, Kwon SW, Hong SJ, Chung BH, et al. Translation Validity and Reliability of I-PSS Korean Version. Korean J Urol 1996;37:659-65.

11. Abrams P, Cardozo L, Fall M, Griffiths D, Rosier P, Ulmsten U, et al. The standardisation of terminology of lower urinary tract function: report from the Standardisation Sub-committee of the International Continence Society. Neurourol Urodyn 2002;21:167-78.

12. Schafer W, Abrams P, Liao L, Mattiasson A, Pesce F, Spangberg A, et al. Good urodynamic practices: uroflowmetry, filling cystometry, and pressure-flow studies. Neurourol Urodyn 2002;21:261-74.

13. Griffiths D, Hofner K, van Mastrigt R, Rollema HJ, Spangberg A, Gleason D. Standardization of terminology of lower urinary tract function: pressure-flow studies of voiding, urethral resistance, and urethral obstruction. International Continence Society Subcommittee on Standardization of Terminology of Pressure-Flow Studies. Neurourol Urodyn 1997;16:1-18.

14. Spirtes P, Glymour C, Scheines R. Direct vs. indirect causation. In:
Spirtes P, Glymour C, Scheines R, editors. Causation, prediction, and search. 2nd ed. Cambridge: MIT Press; 2000. p. 42-7.

15. Derksen S, Keselman HJ. Backward, forward and stepwise automated subset selection algorithms: Frequency of obtaining authentic and noise variables. Br J Math Stat Psychol 1992;45:265-82.

16. DeLong ER, DeLong DM, Clarke-Pearson DL. Comparing the areas under two or more correlated receiver operating characteristic curves: a nonparametric approach. Biometrics 1988;44:837-45.

17. Choi SY, Kim TH, Myung SC, Moon YT, Kim KD, Kim YS, et al. Impact of changing trends in medical therapy on surgery for benign prostatic hyperplasia over two decades. Korean J Urol 2012; 53:23-8.

18. Javle P, Jenkins SA, Machin DG, Parsons KF. Grading of benign prostatic obstruction can predict the outcome of transurethral prostatectomy. J Urol 1998;160:1713-7.

19. Gotoh M, Yoshikawa Y, Kondo AS, Kondo A, Ono Y, Ohshima S. Prognostic value of pressure-flow study in surgical treatment of benign prostatic obstruction. World J Urol 1999;17:274-8.

20. Jensen KM, Jorgensen JB, Mogensen P, Bille-Brahe NE. Some clinical aspects of uroflowmetry in elderly males. A population survey. Scand J Urol Nephrol 1986;20:93-9.

21. Scales CD Jr, Voils CI, Fesperman SF, Sur RL, Kubler H, Preminger GM, et al. Barriers to the practice of evidence-based urology. J Urol 2008;179:2345-9.

22. Seki N, Takei M, Yamaguchi A, Naito S. Analysis of prognostic factors regarding the outcome after a transurethral resection for symptomatic benign prostatic enlargement. Neurourol Urodyn 2006;25: 428-32.

23. Schafer W. Analysis of bladder-outlet function with the linearized passive urethral resistance relation, linPURR, and a disease-specific approach for grading obstruction: from complex to simple. World J Urol 1995;13:47-58. 